\title{
Effect of nutrient intakes on anthropometric profiles among university students from a selected private University in Klang Valley, Malaysia
}

\author{
Hui Chin Koo ${ }^{1}$, Hadirah Z2 ${ }^{2}$, Airina A2, Nurul Alifatul Amrina R², Faziela N²
}

\author{
1. Department of Bioscience, Faculty of Applied Sciences, Tunku Abdul Rahman University College, Kuala \\ Lumpur, Malaysia. \\ 2. Department of Healthcare Professional, Faculty of Health and Life Sciences, Management \& Science \\ University, Shah Alam, Malaysia.
}

\begin{abstract}
Background: Dietary composition is the cornerstone of weight management. This study aimed to investigate the effect of nutrient intakes on anthropometric profiles among university students.

Methods: This cross-sectional study was conducted among 411 students aged 18-29 years, purposive sampled from a selected private university in Klang Valley, Malaysia. Anthropometric profiles were measured. Nutrient intakes were assessed by 3-day 24-hour diet recalls.

Results: Respondents on average had adequate macronutrient intakes, however, total consumption of dietary fiber and micronutrients were fell short of recommended levels. Significant negative associations were found between body mass index (BMI) and all the macronutrients, calcium, thiamine, riboflavin and niacin. Body fat percentage was significantly associated with all the macronutrients, calcium, zinc, thiamine and niacin. Significant inverse associations were also found between waist circumference and carbohydrate, fiber, thiamine, riboflavin and niacin. Visceral fat showed significant inverse associations with carbohydrate, fat, fiber, thiamine, riboflavin and niacin. Further, after adjusting for sex, gender and race, BMI was associated with niacin ( $\beta=-$ 0.161, $\mathrm{p}=0.027)$. Body fat percentage was also found significantly associated with niacin $(\beta=-0.180, \mathrm{p}=0.002)$ and riboflavin $(\beta=-0.132, p=0.014)$.
\end{abstract}

Conclusion: Micronutrients, especially B vitamins, are important in weight management among the young adults.

Keywords: Anthropometric measurements, fiber, macronutrients, micronutrients, malaysian university students.

DOI: https://dx.doi.org/10.4314/ahs.v19i2.50

Cite: as: Koo HC, Hadirah Z, Airina A, Nurul Alifatul Amrina R, Faziela N. Effect of nutrient intakes on anthropometric profiles among university students from a selected private university in Klang Valley, Malaysia. Afri Health Sci.2019;19(2): 2243-2251. https:/ / dx.doi.org/ 10.4314/ abs.v19i2.50

\section{Introduction}

Since large scale population data from National Health and Morbidity Survey (NHMS) became available, there

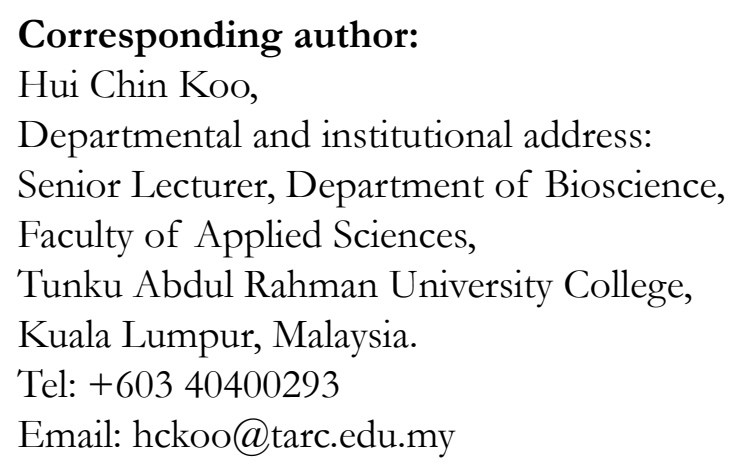

has been a clearly documented dramatic increase in the prevalence of obesity in Malaysia over the past several decades. ${ }^{1}$ Second NHMS was carried out by Ministry of Health in 1996 revealed that $16.6 \%$ and $4.4 \%$ overweight and obese prevalent among the adult aged 18 years and above, respectively. ${ }^{2}$ Nineteen years later, the NHMS V reported that the prevalence of overweight and obese among the adult had dramatically increased to $30.0 \%$ and $17.7 \%$, respectively. ${ }^{3}$ The rise of obesity is not a problem unique to Malaysian. In the global context, obesity has become a major global health challenge, contributing to the rapid growth of several chronic diseases. ${ }^{4}$ This trend is driven by a range of factors especially a remarkable shift from traditional diets towards a high energy density diets, with a greater role for fat and added sugars in foods, and reduced intake of fiber. ${ }^{5}$ 
Poor eating habits is a major public health concern among young adults who experienced transition into university life, during which, they are lack of costs and financial resources, and the availability of convenience meals. ${ }^{6}$ These factors pose a barrier against adoption of healthy eating behaviors. $^{7}$ Although these behaviors of young adults are considered temporary, as part of university life; unhealthy habits picked up at this age generally persist in older adult life and may lead to an array of negative physical changes in the youth and trigger non-communicable diseases in adulthood. ${ }^{8}$ Rapid changes in physical growth and psychosocial development have placed these young adults as nutritionally vulnerable groups with poor eating habits, that fail to meet nutrient requirements. ${ }^{9}$ In this respect, a focus on strengthening protective factors in prevention of obesity by establish healthy eating may have a lasting impact on the health among the young adults, and consequently on the health of their future families. ${ }^{6}$

Dietary composition is the cornerstone of weight management across the life cycle. Effect of macronutrients on weight management has been widely studied and reported in the literature.10 However, weight reduction diets that focus on macronutrient composition should attend to the overall quality of the diet, including the adequacy of micronutrient intakes.11 Multiple micronutrients deficiencies are a worldwide issue, and extend to a range of population groups including young adults.12 Studies from Malaysia13 and other countries12,14 have reported high prevalence of micronutrients deficiencies in young adults, particularly university students, despite they had adequate macronutrient intakes. Micronutrients deficiencies have been observed in obese individuals in many parts of the world, and this issue may influence several physiological body functions and increase the risk of co-morbidities.11 Unfortunately, none as far as is known, had thoroughly investigated the effect of nutrient intakes, particularly micronutrients on anthropometric measurements among the Malaysian university students.

Further with regard to the above, exploring and understanding the potential dietary factors contributing to the emerging obesity epidemic, including macronutrients, dietary fiber and micronutrients seem the most promising approach in guiding tailored-made strategies to most effectively tackle obesity problem. Therefore, this study was conducted to explore the nutrient intakes and nutritional status, as well as the effect of nutrient intakes on anthropometric profiles through a selected sample of private university students in Klang Valley, Malaysia.

\section{Methods}

A cross-sectional study was conducted from October 2016 to April 2017 involving Malaysian university students aged 18 to 29 years from a private university in Klang Valley, by purposive sampling method. Using Krejcie and Morgan (1970)'s formulation, ${ }^{15}$ the estimated sample was based on a total of 15,000 university students aged 18 to 29 years, a $95 \%$ confidence level, a relative precision of $5 \%$ and predicted prevalence of $50 \%$. The estimated sample was 375; however, after adjusting for the $20 \%$ drop out rate, a sample of 450 was required to meet the criteria. Ultimately, data from 411 students were analyzed. Students were eligible for inclusion in the study if they were: full time registered Malaysian university student, male or female, from all ethnic groups (Malay, Chinese, Indian, Bumiputra Sabah, bumiputra Sarawak and other bumiputra), aged 18 to 29 years and not pregnant. Students with bedridden or physical disability were excluded.

Ethical approval was received from the Management and Science University research ethics committee prior to data collection. Students were provided with an information sheet. Written consent was obtained from the student before the study began, in order to enable us to administer the three days 24-hour diet-recall interview and do anthropometric measurements. Socio-demographic data such as sex, age and race of the students were collected using a socio-demographic questionnaire.

The body weight of each student was measured twice using a calibrated TANITA digital scale Model HD-314 (Tanita Co., Tokyo, Japan) and recorded to the nearest $0.1 \mathrm{~kg}$. The students were weighed with their cloth on, barefooted, without belts and empty pockets. Students were asked to stand still with the body weight equally distributed on both feet. The angles of head of the respondents had to be in the Frankfurt plane, where the body was upright, upper limbs were at the body's sides with the palms facing forward, feet flat and directed forward, directly facing the researcher. The height of the student was measured twice using a SECA portable stadiometer 
Model 217 (SECA GmbH \& Co., Hamburg, Germany) attached to a smooth wall and recorded to the nearest $0.1 \mathrm{~cm}$. The respondents were asked to stand erect, barefooted, with heels, buttocks, head and shoulder blades in a vertical line against the wall. Height was measured in the upright position against a vertical scale and with the head positioned so that the top of the external auditory meatus was in the level with the inferior margin of the bone orbit. Body mass index (BMI) for Asian classification was based on World Health Organization (WHO) guideline. ${ }^{16}$

The percentage of body fat and visceral fat were measured twice by bioelectrical impedance using the TANITA digital scale Model HD-314 (Tanita Co., Tokyo, Japan) and nearest to $0.1 \%$. The students stood bare footed on the scale, after the electrode of the scale was cleaned to remove any debris. Any objects that could interfere with the readings were removed from the pockets before the measurements were taken.

Waist circumference (WC) of the students was measured twice, according to the standardized protocol by the National Health and Nutrition Examination Survey (NHANES), which assessed by the WC just above the right iliac crest at the mid-axillary line. The WC was measured to the nearest $0.1 \mathrm{~cm}$ by using a Lufkin tape model W606PM (Apex Tool Group, Maryland, USA). The reported values of all the anthropometric measurements were the average values from the two readings taken.

The nutrient intakes were assessed using three non-consecutive days' 24-hour diet recalls, consisting of 2 weekdays and 1 weekend day over the period of a week. They were completed through face-to-face interviews with each student using household measurements, portion sizes and estimated weights of the food consumed. Tableware items such as dishes, spoons, bowls and glasses in commonly-used sized, food model and pictures of common foods were used to assess the food intake to enhance the portion sizes and the estimation of their respective weights. The information received was carefully checked in order to avoid omitted or misreported data. Macronutrients, dietary fiber and micronutrients were determined from the 3-day 24-hour diet recalls using Nutritionist Pro software (Axxya Systems, United States), based princi- pally on the Nutrient Composition on Malaysian Food and the food product labels. The students' intakes were compared with the Malaysian Recommended Nutrient Intakes (RNI). ${ }^{17}$

A comparison of energy intake with estimated basal metabolic rate (BMR) was used to estimate the number of students who might be under-reported or over-reported their energy intake. Estimated of BMR had been derived from standard equations based on age and sex reported by Ismail et al (1998)18. For young adult in a non-dieting population, Torun et al. (1996)19 suggested that a ratio between energy intake and BMR of less than 1.39 for male and 1.30 for female; and more than 2.24 and 2.10 for female, were considered under-reported and over-reported, respectively. Students who under-reported or over-reported their energy intake were excluded from the analyses which involved dietary intake.

SPSS (version 22.0) (IBM SPSS Statistics, 2014) was used for data analysis. Data was entered, cleaned and checked before data analysis. Descriptive statistics were computed for students' demographic information, body mass index (BMI), percentage of fat, visceral fat, waist circumference and intakes of the following nutrients: carbohydrate, protein, fat, dietary fiber, calcium, zinc, vitamin C, thiamine, riboflavin, niacin and folic acid. Nutrient intakes were adjusted for total energy according to the residual adjustment method. ${ }^{20}$ Each variable was examined to ensure the normally distribution using Kolmogorov Smirnov test. The significance of differences between the groups was tested by using chi square and Mann-Whitney $U$ test. Spearman's partial correlation coefficients were conducted to determine associations between anthropometric measurement and nutrient intakes, after controlled for the effect of energy intake. The stepwise linear regression analyses were conducted to explore whether nutrient intakes were related to anthropometric measurements independently of confounding factors. Before performing stepwise linear regression, all the model assumptions including the linear relationship, multivariate normality, independent errors, outliers, multicollinearity and homoscedasticity were run and fulfilled. The variables that approached $\mathrm{p}<0.25$ or achieved significance $\mathrm{p}<0.05$ in the simple linear regression analyses were selected as confounders, which including sex, gender and age. 


\section{Results}

The demographic information, anthropometric profiles and prevalence of under- and over-reported of energy intake among students are presented in Table 1. The median age of the students was 21 (IqR 3) years. Out of 411 students, more than half of the proportion was female (62.3\%). A majority of the students were Malay (69.6\%). The median BMI, body fat, visceral fat and waist circumference of the students were 23.0 (IqR 6.9 ) $\mathrm{kg} / \mathrm{m}^{2}, 29.7$ (IqR 10.6)\%, 4.5 (IqR 5.5) and 76.0 (IqR 17.0) cm, respec- tively. A majority of them were normal weight (43.8\%), followed by overweight (29.0\%), obese (20.0\%) and underweight $(6.3 \%)$. Male showed significantly higher visceral fat $(p<0.001)$ and waist circumference $(p<0.001)$ as compared to female. Percentage of fat was significantly higher in female $(\mathrm{p}<0.001)$. Out of 411 students, a total of $8.3 \% \quad(n=34)$ were under-reported their energy intake; meanwhile, prevalence of over-reported energy intake was $1.5 \%(n=6)$. Students who under-reported or over-reported their energy intake were excluded from the analyses which involved dietary intake.

Table 1: Demographic characteristics, anthropometric profiles and prevalence of under- and over-reported of energy intake among students $(n=411)$

\begin{tabular}{llll}
\hline Variables & Total (n=411) & Male (n=155) & Female (n=256) \\
\cline { 2 - 4 } & Median (IqR) & Median (IqR) & Median (IqR) \\
\hline Age (years) & $21.0(3.0)$ & $22.0(3.0)$ & $21.0(3.0)$ \\
Race[n(\%)] & & & \\
Malay & $286(69.6)$ & $111(71.6)$ & $175(68.4)$ \\
Indian & $95(23.1)$ & $35(22.6)$ & $60(23.4)$ \\
Chinese & $26(6.3)$ & $8(5.2)$ & $18(7.0)$ \\
Others & $4(1.0)$ & $1(0.6)$ & $3(1.2)$ \\
Anthropometric & & & \\
measurements & & $23.3(7.3)$ & $22.7(6.9)$ \\
Body mass index (kg/m $\left.{ }^{2}\right)$ & $23.0(6.9)$ & $21.7(11.1)^{\dagger}$ & $32.1(7.6)$ \\
Percentage of body fat (\%) & $29.7(10.6)$ & $6.0(7.5)^{\dagger \dagger}$ & $4.0(4.5)$ \\
Visceral fat & $4.5(5.5)$ & $80.0(15.0)^{\dagger \dagger}$ & $74.0(14.0)$ \\
Waist circumference (cm) & $76.0(17.0)$ & & \\
Nutritional status [n(\%)] & & $8(5.2)$ & $18(7.0)$ \\
Underweight & $26(6.3)$ & $66(42.6)$ & $114(44.5)$ \\
Normal weight & $180(43.8)$ & $44(28.4)$ & $75(29.3)$ \\
Overweight & $119(29.0)$ & $37(23.8)$ & $49(19.2)$ \\
Obese & $86(20.9)$ & & $21(8.2)$ \\
Under- or over-reporting of \\
energy intake [n(\%)]
\end{tabular}

Significantly different between boys and girls: ${ }^{\dagger} \mathrm{U}=5866, \mathrm{z}=-11.973, p<0.001 ;{ }^{\dagger \dagger} \mathrm{U}=13428, \mathrm{z}=-5.500$, $p<0.001 ;{ }^{\dagger \dagger} \mathrm{U}=12842, \mathrm{z}=-5.998, p<0.001$

The overall dietary intake obtained by 3-day 24-hour dietary recalls is presented in Table 2 . Overall, students on average had an adequate protein intake according to Malaysian RNI. ${ }^{17}$ However, consumption of dietary fiber was low with the Malaysian $\mathrm{RNI}^{17}$ achievement $11.0 \%$ in both sexes. Besides, it was noteworthy that the levels shown below the Malaysian $\mathrm{RNI}^{17}$ for energy and all the micronutrients including calcium, zinc, vitamin $\mathrm{C}$, thiamine, riboflavin, niacin and folic acid. Energy, all the macronutrients, calcium, zinc and niacin intakes tended to be significantly higher among male. Females on the other hand had significantly greater vitamin $\mathrm{C}$ consumption. 
Table 2. Overall nutrient intakes obtained by three-day 24-hour dietary recalls of the respondents $(\mathrm{n}=371)$

\begin{tabular}{|c|c|c|c|c|c|c|}
\hline \multirow[t]{2}{*}{ Nutrient intakes } & \multirow[t]{2}{*}{ Sex } & \multirow[t]{2}{*}{ Mean } & \multirow[t]{2}{*}{ SD } & \multicolumn{2}{|l|}{ Range } & \multirow[t]{2}{*}{ RNI (\%) } \\
\hline & & & & Min & $\operatorname{Max}$ & \\
\hline \multirow[t]{2}{*}{ Energy $(\mathrm{g}) * * *$} & Male $(n=155)$ & 2280 & 48.2 & 1812 & 2988 & 93.4 \\
\hline & Female $(n=256)$ & 1984 & 76.8 & 1534 & 2657 & 99.2 \\
\hline \multirow[t]{2}{*}{ Protein $(\mathrm{g})^{* * *}$} & Male $(n=155)$ & 65.8 & 30.0 & 16.3 & 136.3 & 106.1 \\
\hline & Female $(n=256)$ & 57.9 & 57.6 & 12.4 & 130.6 & 105.3 \\
\hline \multirow[t]{2}{*}{ Carbohydrate $(\mathrm{g})^{*}$} & Male $(n=155)$ & 235.4 & 74.2 & 172.3 & 289.0 & - \\
\hline & Female $(n=256)$ & 212.7 & 64.2 & 145.5 & 280.6 & \\
\hline \multirow[t]{2}{*}{ Fat $(\mathrm{g})^{* *}$} & Male $(n=155)$ & 62.1 & 26.7 & 13.8 & 205.9 & - \\
\hline & Female $(n=256)$ & 54.5 & 23.5 & 16.8 & 157.3 & \\
\hline \multirow[t]{2}{*}{ Dietary fibre (g) } & Male $(n=155)$ & 2.2 & 1.8 & 0 & 10.7 & 11.0 \\
\hline & Female $(n=256)$ & 2.2 & 1.5 & 0 & 8.0 & 11.0 \\
\hline \multirow[t]{2}{*}{ Calcium (mg)* } & Male $(n=155)$ & 412.2 & 213.3 & 17.5 & 1148.0 & 51.5 \\
\hline & Female $(n=256)$ & 370.6 & 201.2 & 47.4 & 1230.4 & 46.3 \\
\hline \multirow[t]{2}{*}{ Zinc $(m g)^{* *}$} & Male $(\mathrm{n}=155)$ & 5.2 & 3.5 & 0.2 & 27.8 & 77.6 \\
\hline & Female $(n=256)$ & 4.2 & 2.4 & 0 & 15.5 & 85.7 \\
\hline \multirow[t]{2}{*}{ Vitamin C $(\mathrm{mg})^{* *}$} & Male $(n=155)$ & 19.7 & 22.8 & 0.13 & 130.0 & 28.1 \\
\hline & Female $(n=256)$ & 25.4 & 28.5 & 0 & 226.1 & 36.3 \\
\hline \multirow[t]{2}{*}{ Thiamine (mg) } & Male $(n=155)$ & 0.7 & 0.5 & 0.1 & 2.3 & 58.3 \\
\hline & Female $(n=256)$ & 0.8 & 0.9 & 0.1 & 1.9 & 72.7 \\
\hline \multirow[t]{2}{*}{ Riboflavin (mg) } & Male $(n=155)$ & 1.2 & 1.1 & 0.2 & 12.0 & 92.3 \\
\hline & Female $(n=256)$ & 1.1 & 0.8 & 0.2 & 9.0 & 100 \\
\hline \multirow[t]{2}{*}{$\operatorname{Niacin}(\mathrm{mg}) * *$} & Male $(n=155)$ & 11.7 & 5.7 & 2.1 & 35.5 & 73.1 \\
\hline & Female $(n=256)$ & 10.2 & 4.9 & 0.7 & 32.9 & 72.9 \\
\hline \multirow[t]{2}{*}{ Folate $(\mu \mathrm{g})$} & Male $(\mathrm{n}=155)$ & 59.6 & 42.0 & 2.3 & 343.7 & 14.9 \\
\hline & Female $(n=256)$ & 53.6 & 39.2 & 0 & 349.2 & 13.4 \\
\hline
\end{tabular}

RNI: Recommended Nutrient Intakes for Malaysia 2005; significantly different between male and female*p<0.05;**p<0.01; $* * * p<0.001$

Significant negative associations were shown between $\mathrm{BMI}$ and all the macronutrients and B vitamins (thiamine, riboflavin and niacin). Body fat percentage was inversely associated with all the macronutrients, zinc, calcium, thiamine and niacin. Significant negative associations were found between waist circumference and carbohydrate, dietary fiber and B vitamins (thiamin, riboflavin and niacin). Visceral fat was inversely associated with carbohydrate, fat, dietary fiber and $\mathrm{B}$ vitamins (thiamine, riboflavin and niacin). The findings from the simple linear regression analyses were further explored using stepwise linear regressions, with sex, race and age adjusted. The outcomes are presented in Table 3. Inverse associations were found between niacin and BMI, as well as body fat percentage, after confounder adjusted. A standard deviation (SD) increased in niacin, lead to 0.161 SD and 0.180 SD decreased in BMI and body fat percentage, respectively. Body fat percentage was inversely associated with riboflavin too. A standard deviation increased in riboflavin, lead to $0.132 \mathrm{SD}$ decreased in body fat percentage. 
Table 3. Summary of stepwise linear regression for nutrient intakes predicting

various anthropometric measurements $(\mathrm{n}=\mathbf{3 7 1})$

\begin{tabular}{|c|c|c|c|c|c|c|c|c|}
\hline \multirow[t]{2}{*}{$\begin{array}{l}\text { Nutrient } \\
\text { intakes }\end{array}$} & \multirow{2}{*}{$\begin{array}{l}\text { Body mass } \\
\text { index }^{\dagger} \\
\text { Standardiz } \\
\text { ed } \beta\end{array}$} & \multicolumn{2}{|c|}{$\begin{array}{l}\text { Body fat } \\
\text { percentage }^{\dagger \dagger}\end{array}$} & \multicolumn{3}{|c|}{ Waist circumference $^{i \hbar t}$} & \multicolumn{2}{|c|}{ Visceral fat } \\
\hline & & $p$-value & $\begin{array}{l}\text { Standardi } \\
\text { zed } \beta\end{array}$ & $p$-value & $\begin{array}{l}\text { Standard } \\
\text { ized } \beta\end{array}$ & $\begin{array}{l}p- \\
\text { value }\end{array}$ & Standardized $\beta$ & $p$-value \\
\hline Protein & 0.056 & 0.279 & 0.015 & 0.709 & 0.026 & 0.600 & 0.045 & 0.901 \\
\hline $\begin{array}{l}\text { Carbohydr } \\
\text { ate }\end{array}$ & -0.101 & 0.134 & -0.064 & 0.234 & -0.113 & 0.080 & -0.099 & 0.135 \\
\hline Fat & -0.040 & 0.541 & 0.056 & 0.286 & -0.042 & 0.504 & -0.041 & 0.526 \\
\hline Fiber & -0.042 & 0.492 & -0.031 & 0.520 & -0.080 & 0.177 & -0.040 & 0.503 \\
\hline Calcium & -0.043 & 0.505 & -0.087 & 0.091 & -0.015 & 0.806 & -0.028 & 0.662 \\
\hline Zinc & -0.006 & 0.919 & 0.023 & 0.610 & 0.048 & 0.385 & -0.005 & 0.928 \\
\hline Vitamin C & 0.037 & 0.526 & 0.003 & 0.954 & 0.025 & 0.664 & 0.040 & 0.489 \\
\hline Thiamin & -0.020 & 0.680 & -0.006 & 0.882 & -0.028 & 0.552 & -0.016 & 0.739 \\
\hline Riboflavin & 0.115 & 0.089 & -0.132 & $0.014^{*}$ & 0.094 & 0.145 & 0.118 & 0.073 \\
\hline Niacin & -0.161 & $0.027^{*}$ & -0.180 & $0.002 * *$ & -0.134 & 0.054 & -0.131 & 0.065 \\
\hline Folate & 0.101 & 0.074 & 0.030 & 0.500 & 0.091 & 0.092 & 0.083 & 0.135 \\
\hline
\end{tabular}

\footnotetext{
Adjusted regression coefficient for age, sex and race; Nutrient intakes were adjusted for energy according to the residual adjustment method (Willet et al., 1997) and included in the models as residual value; Model assumptions were fulfilled; There were no interactions among independent variables; No multicollinearity detected; ${ }^{\dagger}$ Coefficient of determination $\left(\mathrm{R}^{2}\right)=0.271 ;{ }^{\dagger \dagger}$ Coefficient of determination $\left(\mathrm{R}^{2}\right)=0.645 ;{ }^{i \dagger}$ Coefficient of determination $\left(\mathrm{R}^{2}\right)=0.386 ;{ }^{i \dagger \dagger}$ Coefficient of determination $\left(\mathrm{R}^{2}\right)=0.341$.
}

\section{Discussion}

This is the first study to investigate the effect between nutrient intakes and anthropometric profiles among the Malaysian university students. Our study reveals the significant inverse associations between nutrient intakes and anthropometric measurements among the students. The present study also shows that students on average had adequate macronutrient intakes, however, total consumption of dietary fiber and micronutrients fell short of recommended levels.

In the present study, when the overweight group and the obese group were combined, they account for $49.9 \%$ of the study population, which was much higher than the Malaysian university students from other states. ${ }^{13,21}$ The differences in prevalence of overweight/obesity can be explained by the use of BMI classification guideline, where the present study applied Asian BMI classification from WHO (2004) ${ }^{16}$ but others applied general BMI classification from WHO (2000). ${ }^{22}$ Males showed significantly higher visceral fat and waist circumference, but female had higher body fat percentage. Sex differences in adipose tissue distribution are well-supported by many literature findings. Women generally have higher adiposity relative to men throughout the entire lifespan, and they store more fat in the gluteal-femoral region, whereas men store more fat in the visceral and abdominal depot. ${ }^{23}$
Overall, the dietary intakes of the students were not compatible with that recommended by the Malaysian RNI, especially for fiber and all the micronutrient intakes. In the present study, despite adequate carbohydrates consumption, mean intake of fiber well below recommended level, and may be considered insufficient for optimal health promotion and chronic disease prevention. ${ }^{24}$ This was apparently due to low consumption of vegetables and fresh fruits as observed in the 3-day 24-hour diet recall data of the students and possibly due to inadequate quantities of other sources of fiber such as legumes, whole grains and grain-based products.

Micronutrients, including vitamins and minerals, are required in small amounts and interact with one another to regulate physiological functions. ${ }^{25}$ However, micronutrient intakes in the present study were fell short of recommended levels. This trend is similar to other studies where young adults tended to have inadequate micronutrient intakes, including calcium, zinc, folate, vitamin C, thiamin, riboflavin, niacin. ${ }^{12}$ This was apparently due to low consumption of fruits and vegetables, whole grain, milk and dairy products among the young adults, especially university students. ${ }^{26}$

Significant negative associations were found between anthropometric measurements and macronutrients, fiber 
and micronutrients. These findings are well-supported by several literature findings. ${ }^{11,27}$ During the past several years, evidence has been raised that obesity can be associated with substantial nutrient deficiencies.11Macronutrient composition has been suggested as an important determinant in the obesity epidemic, by exhibiting a hierarchical effect on short-term food intake suppression and satiety; promote satiety may eventually facilitate weight loss through reduced energy consumption. ${ }^{28} \mathrm{Be}-$ sides macronutrients, fiber showed negative associations with several anthropometric measurements too. High fiber intake was associated with altering of gastrointestinal myoelectrical activity, decrease accessibility of substrates to $\alpha$-amylase, delay gastric emptying, decrease glucose diffusion through the water layer and increase in satiety. ${ }^{24}$

Micronutrient deficiencies may contribute to fat deposition and chronic inflammation, impair the immune system, influence several physiological body functions and increase the risk of co-morbidities. ${ }^{29}$ In the present study, body fat percentage was associated with riboflavin and niacin after adjusting for sex, gender and race. On the other hand, niacin also associated with BMI after potential confounders adjusted. These findings are in accordance with several studies. ${ }^{30,31}$ A number of possible mechanisms may underlie our findings of association between the B vitamins and adiposity. Study has shown that the cofactor flavin adenine dinucleotide that includes riboflavin as a central component epigenetically regulates energy-expenditure adipocyte genes. ${ }^{32}$ Further, research demonstrated that niacin treatment attenuates obesity-induced adipose tissue inflammation through increased adiponectin and anti-inflammatory cytokine expression and reduced pro-inflammatory cytokine expression in a niacin receptor-dependent manner. ${ }^{30}$ Findings from previous experiment also suggested that niacin exerted beneficial effect on adiposity, insulin sensitivity, plasma lipids and that it specifically modulated the level of serum adiponectin under obese condition. ${ }^{31}$ The outcomes of this present study could have important implications for the development of interventions that more effectively reduce obesity among young adults, especially university students in Klang Valley, Malaysia, such as introduce meals that high in fiber and micronutrients.

We acknowledge that our study has some limitations. Evaluation of nutritional intake has some methodolog- ical weakness. To address this limitation, under-reported or over-reported data were excluded from the analyses which involved dietary intake. Besides, the total micronutrient intakes could be probably underestimated since we had calculated the average intake of vitamins and minerals from all sources of the foods, without considering fortified foods or medication which students mightbe consumed. Students who participated in the present study were more likely to be homogeneous in terms of their background diet. This homogeneity of students in the present study regarding their background diet could limit the generalisability of our findings.

Despite these limitations, the present study has several strengths. We used the Malaysian $\mathrm{RNI}^{17}$ to estimate nutrient intake adequacy. The use of RNI as a reference for estimating nutritional adequacy would not lead to an overestimation of the prevalence of nutrient inadequacy. Besides, nutrient intakes were adjusted for total energy according to the residual adjustment method. An advantage of this method over others is that it provides a measure of nutrient intakes that is independent of total energy intake. ${ }^{18}$ We were able to adjust for numerous potential confounders too.

\section{Conclusion}

In light of the escalating prevalence rates of obesity, governments and healthcare organizations are endeavoring to develop and implement appropriate healthy balance diet interventions to manage devastating of obesity. This study presents vital information on the nutrient intakes among university students in a selected private university in Malaysia, and their associations with anthropometric profiles. Students on average had adequate macronutrient intakes; however, total consumption of dietary fiber and micronutrients were fell short of recommended levels. Macronutrients, fiber and micronutrients, especially B vitamins, may improve the anthropometric measurements. The findings of this study highlight the importance of focusing on these nutrients in weight management among young adults, especially university students.

\section{Acknowledgments}

We extend our gratitude to Management and Science University, for providing us with the help throughout data collection and facilities. Heartily thank to the university students for participating. 


\section{Declaration of conflict of interest}

All authors declare that they have no conflict of interest.

\section{References}

1. Lim KG. A review of adult obesityresearch in Malaysia. Med J of Malaysia. 2016, 71(Suppl 1): 1-19.

2. Institute of Public Health (IPH). National Health and Morbidity Survey (NHMS) (1996) Non-communicable diseases, Ministry of Health Malaysia. Vol II.

3. Institute of Public Health (IPH). National Health and Morbidity Survey (NHMS) (2015) Non-communicable diseases, Ministry of Health Malaysia. Vol V.

4. Ng M, Fleming T, Robinson M et al. Global, regional, and national prevalence of overweight and obesity in children and adults during 1980-2013: a systematic analysis for the Global Burden of Disease Study. Lancet 2014, 384: 766-781.

5. Wang Y, Wang L, Xue $\mathrm{H}$ et al. A review of the growth of the fast food industry in China and its potential impact of obesity. Int J of Environ Res Publ Health. 2016, 13: 11121127.

6. El-Kassas G, Ziade F. Exploration of the dietary and lifestyle behaviors and weight status and their self-perceptions among health sciences university students in North Lebanon. BioMed Res Int, 2016, 2016: 1-14.

7. Al-Nakeeb Y, Lyons M, Dodd LJ, Al-Nuaim A. An investigation into the lifestyle, health habits and risk factors of young adults. Int J Environ Res Publ Health. 2015, 12(4): 4380-4394.

8. World Health Organization. 2011. Global status report on non-communicable disease 2010. Geneva, Switzerland.

9. World Health Organization. 2013. Global action plan for the prevention and control of non-communicable disease 2013-2020. Geneva, Switzerland.

10. Ebbeling CB, Swain JF, Feldman HA. Effects of dietary composition on energy expenditure during weightloss maintenance. JAMA. 2012, 307(24): 2627-2634.

11. Garcia OP, Ronquillo D, Caanamo Mdel C et al. Zinc, iron and vitamins $\mathrm{A}, \mathrm{C}$ and $\mathrm{E}$ are associated with obesity, inflammation, lipid profile and insulin resistance in Mexican school-aged children. Nutrients. 2013, 5: 5012-5030.

12. Fayet-Moore F, Petocz P, Samman S. Micronutrient status in female university students: iron, zinc, copper, selenium, vitamin B12 and folate. Nutrients. 2014, 6: 51035116.

13. Huda N, Ruzita A. Preliminary survey on BMI profile among USM main campus students. PJN 2011, 9: $125-$ 127.

14. Hwalla N, Dhaheri ASA, Radwan $\mathrm{H}$ et al. The prevalence of micronutrient deficiencies and inadequacies in the Middle East and approaches to intervention. Nutrients. 2017, 9: 229-240.

15. Krejcie RV, Morgan DW. Determine sample size for research activities, educational and psychological measurement 1970, 30: 607-610.

16. World Health Organization. 2004. Expert consultation appropriate bodymass index for Asian populations and its implications for policy and intervention strategies. Lancet. 363(9403): 157-163.

17. National Coordinating Committee on Food and Nutrition (NCFFN). Recommended Nutrient Intakes for Malaysia. 2005: Ministry of Health Malaysia, Kuala Lumpur. http://www2.moh.gov.my/images/gallery/rni/insert.pdf.

18. Ismail $\mathrm{MN}, \mathrm{Ng} \mathrm{KK}$, Chee $\mathrm{SS}$ et al. Predictive equations for the estimation of basal metabolic rate in Malaysian adults. Mal J Nutr. 1998, 4: 81-90.

19. Torun B, Davies PSW, Livingstone MBE et al. Energy requirement and dietary energy recommendation for children and adolescents 1 to 18 years old. Eur J Clin Nutr. 1996, 50: S37-S81

20. Willett WC, Howe GR, Kushi LH. Adjustment for total energy intake in epidemiologic studies. Am J Clin Nutr. 1997, 65(suppl): 1220S-1228S.

21. Gopalakrishnan S, Ganeshkumar P, Prakash MVS et al. Prevalence of overweight/obesity among the medical students, Malaysia. Med J Malaysia. 2012, 67 (4): 442-444.

22. World Health Organization. 2000. Obesity: preventing and managing global epidemic. WHO Technical report Series 894. World Health Organization, Geneva

23. White UA, Yourka D, Tchoukalova. Sex dimorphism and depot differences in adipose tissue function. Biochimica et Biophysica Acta. 2014, 1842(2014): 377-392.

24. Slavin J, Tucker M, Cynthia $\mathrm{H}$ et al. Whole grains: definition, dietary recommendations and health benefits. CFW. 2013, 58(4): 191-198.

25. Kreider RB, Wilborn CD, Taylor L et al. ISSN exercise \& sport nutrition review: research \& recommendations. JISSN. 2010, 7: 1-7.

26. Poddar KH, Hosig KW, Nickols-Richardson SM et al. Low-fat dairy intake and body weight and composition changes in college students. J Am Diet Assoc. 2009, 109(8):1433-1438. 
27. Gunanti IR, Marks GC, Al-Mamun A et al. Low serum vitamin B-12 and folate concentrations and low thiamin and riboflavin intakes are inversely associatedwith greater adiposity in Mexican American children. $J$ Nutr. 2014, 144(12): 2027-2033.

28. Austin GL, Ogden LG, Hill JO. Trends in carbohydrate, fat, and protein intakes and association with energy intake in normal-weight, overweight, and obese individuals: 1971-2006. Am J Clin Nutr. 2011, 93: 836-843.

29. Zavala G, Long KZ, Garcia OP et al. Specific micronutrient concentrations are associated with inflammatory cytokines in a rural population of Mexican women with a high prevalence of obesity. BrJ Nutr. 2013, 109(4): 686694.

30. Wanders D, Graff EC, White BD et al. Niacin increases adiponectin and decreased adipose tissue inflammation in high fat diet-fed mice. Plos one. 2013, Doi: 10.1371/journal.pone.0071285.

31. Morakinyo AO, Samuel TA, Adekunbi DA et al. Niacin improves adiponectin secretion, glucose tolerance and insulin sensitivity in diet-induced obese rats. EJBAS, 2015, 2(4): 261-267.

32. Hino S, Sakamoto A, Nagaoka K et al. FAD-dependent lysine-specific demethylase-1 regulates cellular energy expenditure. Nat Commu. 2012, 3: 758-770. 